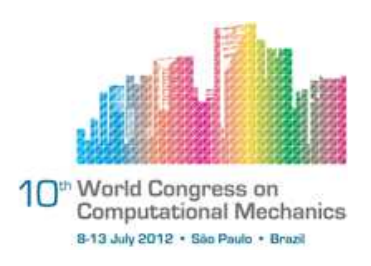

\title{
SPECTRAL ELEMENT APPROXIMATION OF FREDHOLM INTEGRAL EIGENVALUE PROBLEMS
}

\author{
S. P. Oliveira ${ }^{1}$ \\ ${ }^{1}$ Department of Mathematics, Federal University of Paraná (saulopo@ufpr.br)
}

\begin{abstract}
The Karhunen-Loève expansion of a Gaussian process, a common tool on finite element methods for differential equations with stochastic coefficients, is based on the spectral decomposition of its covariance function. The eigenpairs of the covariance are expressed as a Fredholm integral equation of second kind, which can be readily approximated with piecewise-constant finite elements. In this work, the spectral element method with GaussLobatto-Legendre (GLL) collocation points is employed to approximate this eigenvalue problem. Similarly to piecewise-constant finite elements, this approach is simple to implement and does not lead to generalized discrete eigenvalue problems (considering that the numerical integration is also performed with GLL points), with the additional advantage of providing high-order approximations of the eigenfunctions. Numerical experiments involving covariance functions in one- and two-dimensional domains illustrate the effectiveness of this approach.
\end{abstract}

Keywords: Spectral element method, Fredholm integral of second kind, Karhunen-Loève expansion.

\section{INTRODUCTION}

Spectral element methods are high-order finite element methods that employ piecewise continuous Lagrange shape functions whose collocation points are roots of orthogonal polynomials. This class of methods has been successful on wave propagation problems mainly because they are flexible to deal with complex geometries and produce low dispersion error [6]. This work considers the use of these methods in the solution of the Fredholm integral equation

$$
\int_{D} C(\mathbf{x}, \mathbf{y}) \phi_{k}(\mathbf{y}) d \mathbf{y}=\lambda_{k} \phi_{k}(\mathbf{x}), \quad k=1,2, \ldots
$$

If the covariance kernel $C$ is symmetric and positive definite, there is a monotonically decreasing set of eigenvalues and an orthonormal set of eigenfunctions satisfying (1). The solution of the eigenvalue problem (1) is relevant to the study of differential equations with random input data. Indeed, if $Z=Z(\mathbf{x}, \omega)$ is a Gaussian random variable with mean $\mu$ and 
positive definite covariance $C$, then $Z$ admits a Karhunen-Loève decomposition:

$$
Z(\mathbf{x}, \omega)=\mu(\mathbf{x})+\sum_{k=1}^{\infty} \sqrt{\lambda_{k}} \phi_{k}(\mathbf{x}) \xi_{k}(\omega)
$$

where $\xi_{k}$ are independent Gaussian random variables with zero mean and unitary variance.

Several approaches to numerically solve (1) are available in the literature. For instance, one can employ the piecewise-constant finite element method [4] or use wavelet bases [3]. Spectral elements methods have been used in the context of integral equations (see [7] and the references therein), but to the author's knowledge they have not yet been explored on the Fredholm integral eigenvalue problem.

The next section reviews the piecewise-constant finite element method for (1) according to [1], and points out some computational issues. Section 4 introduces the spectral element method with Gauss-Lobatto-Legendre collocation points. Numerical experiments are presented on Section 5.

\section{PIECEWISE CONSTANT APPROXIMATION}

The weak form of (1) is given as

$$
\int_{D} \int_{D} C(\mathbf{x}, \mathbf{y}) \phi_{k}(\mathbf{y}) v(\mathbf{x}) d \mathbf{y} d \mathbf{x}=\lambda_{k} \int_{D} \phi_{k}(\mathbf{x}) v(\mathbf{x}) d \mathbf{x} \quad \forall v \in L^{2}(D) .
$$

Let $V_{h}=\operatorname{span}\left\{v_{1}, \ldots v_{n}\right\} \subset L^{2}(D)$. The Galerkin approximation to (3) in $V_{h}$ consists of finding $\lambda_{k}^{h} \in \mathbb{R}$ and $\phi_{k}^{h}(\mathbf{x}) \in V_{h}(1 \leq k \leq n)$ such that

$$
\sum_{j=1}^{n} \phi_{n, j} \int_{D} \int_{D} C(\mathbf{x}, \mathbf{y}) v_{j}(\mathbf{y}) v_{i}(\mathbf{x}) d \mathbf{x} d \mathbf{y}=\lambda_{k}^{h} \sum_{j=1}^{n} \phi_{n, j} \int_{D} v_{j}(\mathbf{x}) v_{i}(\mathbf{x}) d \mathbf{x}
$$

which can be written as a generalized eigenvalue problem:

$$
\begin{gathered}
C \boldsymbol{\phi}_{k}=\lambda_{k}^{h} M \boldsymbol{\phi}_{k}, \\
C_{i, j}=\int_{D} \int_{D} C(\mathbf{x}, \mathbf{y}) v_{j}(\mathbf{y}) v_{i}(\mathbf{x}) d \mathbf{x} d \mathbf{y}, \quad M_{i, j}=\int_{D} v_{j}(\mathbf{x}) v_{i}(\mathbf{x}) d \mathbf{x} .
\end{gathered}
$$

Assume $D$ a polygonal domain discretized by a mesh $\mathcal{T}$ of elements $D^{e}\left(1 \leq e \leq N_{e}\right)$ with maximum element length $h>0$ such that $\dot{D}^{e} \cap \dot{D}^{f}=\emptyset$ for $e \neq f$ and

$$
\bar{D}=\bigcup_{j=1}^{N_{e}} \bar{D}^{e}
$$

Let $\mathcal{P}_{0}^{h}$ be the space of piecewise-constant functions defined in $\mathcal{T}$. The shape functions

$$
v_{e}(\mathbf{x})= \begin{cases}\left|D^{e}\right|^{-1 / 2}, & \mathbf{x} \in D^{e} \\ 0, & \mathbf{x} \notin D^{e}, \quad e=1, \ldots, N_{e}\end{cases}
$$

constitute an orthonormal basis for $\mathcal{P}_{0}^{h}$, hence the discrete eigenvalue problem (5) reduces to

$$
C \boldsymbol{\phi}_{k}=\lambda_{k}^{h} \boldsymbol{\phi}_{k}, \quad C_{i, j}=\sqrt{\frac{1}{\left|D^{i}\right|\left|D^{j}\right|}} \int_{D^{i}} \int_{D^{j}} C(\mathbf{x}, \mathbf{y}) d \mathbf{y} d \mathbf{x} .
$$


The integral in (9) can be approximated as $C_{i, j} \approx \sqrt{\left|D^{i}\right|\left|D^{j}\right|} C\left(\overline{\mathbf{x}}^{i}, \overline{\mathbf{y}}^{j}\right)$, where $\overline{\mathbf{x}}^{i}$ is the barycenter of the element $D^{e}$.

The matrix $C$ is typically dense, in order that a large computational effort (both in memory and CPU time) is required to solve the discrete eigenvalue problem (9). Frauenfelder et al [4] propose algorithmic strategies to meet this demand. Moreover, finite elements of degree higher than zero lead to generalized eigenvalue problems (5) in which the matrix $M$ is not necessarily diagonal. In analogy with finite element methods for transient problems, massdiagonal formulations are attractive, and will motivate the choice of the spectral element basis functions in the next section.

\section{SPECTRAL ELEMENT APPROXIMATION}

Let the interval $[0, L]$ be partitioned into $N_{e}$ equally spaced elements of size $h:=1 / N_{e}$ subdivided into $N$ interior subintervals, so that the total number of nodes is $n:=N N_{e}$. By using the element index by $0 \leq e \leq N_{e}-1$ and the local index $0 \leq j \leq N-1$, the global nodal index is given by $I=I(j, e):=j+e N+1$. The grid coordinates are defined by $x_{I}:=\left(e+\zeta_{j}\right) h, \zeta_{j}=\left(\xi_{j}+1\right) / 2$, where $\xi_{j}$ is the $j$-th collocation point in the interval $[-1,1]$.

The Gauss-Lobatto-Legendre (GLL) collocation points are used in this work. The GLL points are found by numerically solving the equation $\left(1-\xi^{2}\right) P_{N}^{\prime}(\xi)=0$, where $P_{N}^{\prime}$ denotes the derivative of the Legendre polynomial of degree $N$. The same collocation points are used for the numerical integration of the elementary matrices, which renders the mass matrix diagonal [5].

For $D=] 0, L\left[\right.$, the shape functions $v_{I}(x)(1 \leq I \leq n)$ are Lagrangian piecewise polynomial functions of degree $N$ satisfying the relation $v_{I}\left(x_{J}\right)=\delta_{I, J}$, which implies that the matrix coefficients $M_{i, j}$ in (6) satisfy $M_{I, J}=0$ if $I \neq J$. Indeed, let $x=x(\xi)$ be the mapping from the $e$-th element to $[-1,1]$. The contribution to $M_{I, J}$ from the $e$-th element is

$$
M_{I, J}^{e}=\frac{h}{2} \int_{-1}^{1} v_{J}(x(\xi)) v_{I}(x(\xi)) d \xi \approx \frac{h}{2} \sum_{l=1}^{N} w_{l} v_{J}\left(x\left(\xi_{l}\right)\right) v_{I}\left(x\left(\xi_{l}\right)\right),
$$

where $w_{l}$ is the $l$-th GLL integration weight. Because $x\left(\xi_{l}\right)=x_{K}$ with $K=l+e N+1$,

$$
M_{I, J}^{e} \approx \frac{h}{2} \sum_{l=1}^{N} w_{l} v_{J}\left(x_{K}\right) v_{I}\left(x_{K}\right)=\left\{\begin{array}{cl}
(h / 2) w_{j}(I), & I=J=j+e N+1, \\
0, & \text { otherwise }
\end{array}\right.
$$

Therefore,

$$
M_{I, J}=\sum_{e=1}^{N_{e}} M_{I, J}^{e}=\tilde{w}_{I} \delta_{I, J}, \quad \tilde{w}_{I}=\tilde{w}_{I(j, e)}=\left\{\begin{aligned}
(h / 2) w_{j}, & 0<j<N, \\
h w_{j}, & j=0, N .
\end{aligned}\right.
$$

Similar calculations apply to matrix $C$, and the resulting discrete eigenvalue system is

$$
C \phi_{k}=\lambda_{k}^{h} M \phi_{k}, \quad M_{I, J}=\tilde{w}_{I} \delta_{I, J}, C_{I, J}=\tilde{w}_{I} \tilde{w}_{J} C\left(x_{I}, x_{J}\right) .
$$

This system can be further reduced to

$$
\tilde{C} \phi_{k}=\lambda_{k}^{h} \phi_{k}, \quad \tilde{C}_{I, J}=\tilde{w}_{J} C\left(x_{I}, x_{J}\right),
$$


which is a straightforward extension of system (9) to high-order polynomials in the 1D case. However, one should keep in mind that the numerical integration in (10) is not exact, since the integrand $v_{I} v_{J}$ is a polynomial of degree $2 N$, whereas the GLL quadrature is exact for polynomial integrands of degree up to $2 N-1$.

The tensor product of the $1 \mathrm{D}$ spectral elements above naturally lead to $2 \mathrm{D}$ and $3 \mathrm{D}$ basis functions on square/cubic meshes (see [6] for more general geometries).

\section{NUMERICAL EXPERIMENTS}

Following [2], let $D=] 0, L[$ and $C(x, y)$ be defined as

$$
C(x, y)=\sigma^{2} \exp (-|x-y| / \eta), \quad \sigma, \eta>0 .
$$

The exact eigenvalues and eigenfunctions associated with $C$ are

$$
\lambda_{i}=\frac{2 \eta \sigma}{\eta^{2} \gamma_{i}^{2}+1}, \quad \phi_{i}(x)=\frac{\eta \gamma_{i} \cos \left(\gamma_{i} x\right)+\sin \left(\gamma_{i} x\right)}{\sqrt{\left(\eta^{2} \gamma_{i}^{2}+1\right) / 2+\eta}},
$$

where the parameters $\gamma_{1}, \gamma_{2}, \ldots$ are roots of the equation $\left(\eta^{2} \gamma^{2}-1\right) \sin (\gamma)=2 \eta \gamma \cos (\gamma)$. For $D=] 0,1[\times] 0,1[$, let $C(\mathbf{x}, \mathbf{y})$ be the separable exponential covariance function

$$
C(\mathbf{x}, \mathbf{y})=\sigma^{2} \exp \left(-\left|x_{1}-y_{1}\right| / \eta-\left|x_{2}-y_{2}\right| / \eta\right) \text {. }
$$

The exact eigenvalues and eigenfunctions associated with $C(\mathbf{x}, \mathbf{y})$ are respectively $\lambda_{k}^{2 D}=\lambda_{i} \lambda_{j}$ and $\phi_{k}^{2 D}\left(x_{1}, x_{2}\right)=\phi_{i}\left(x_{1}\right) \phi_{j}\left(x_{2}\right)$, where $\left\{\lambda_{i}, \phi_{i}\right\}$ are defined in (16) and the index $n=n(i, j)$ is set to arrange the eigenvalues in decreasing order.

In the numerical experiments below, the input parameters are $\sigma=1$ and $\eta=0.1$. Figure 1 contrasts the first 10 eigenvalues of (15) obtained from systems (9) and (14) (with degree $N=4$ ), as well as the 10th eigenfunction computed with these approaches. A mesh of 20 elements were used in both methods. The smoothness of the numerical eigenfunctions approximated by GLL may be useful if these are employed in subsequent finite element computations.
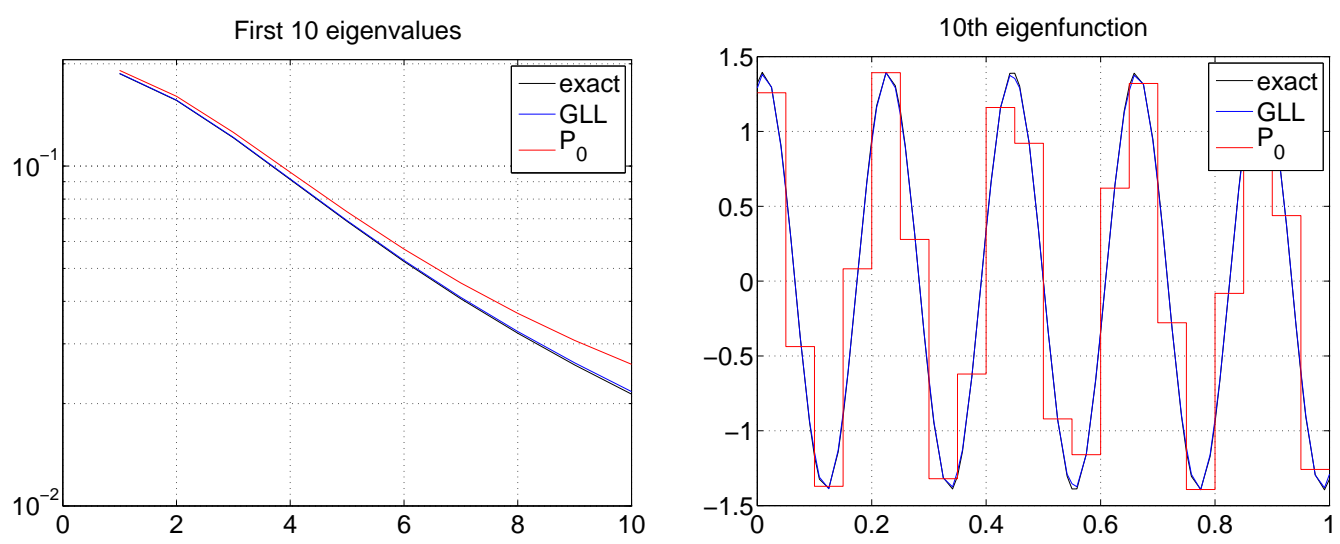

Figure 1. Comparison of eigenvalues and eigenfunctions computed with piecewise constant $\left(P_{0}\right)$ and fourth degree GLL spectral element methods. 
Figure 2 shows the relative error of the first eigenvalue of the covariance kernels (15) and (17) using increasingly refined meshes and GLL elements of degree 1,2, 4 and 8 . The numerical convergence rates did not increase with the polynomial degree, though the regularity of the covariance kernels are low because they depend on $|x-y|$.
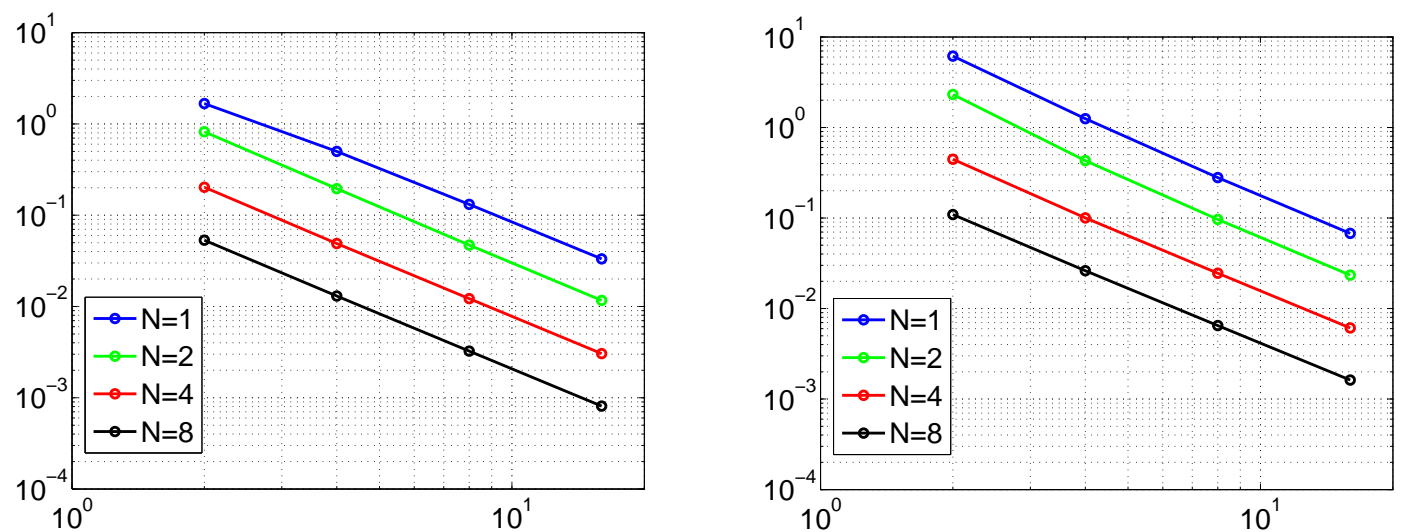

Figure 2. Relative GLL error the first eigenvalue versus number of elements for the 1D (left) and 2D (right) exponential covariance kernel.

In the following we assess the convergence rates of the GLL spectral method for the smooth Gaussian kernel

$$
C(x, y)=\sigma^{2} \exp \left(-(x-y)^{2} / \eta^{2}\right), \quad \sigma, \eta>0 .
$$

For such a kernel a reference solution was calculated with the GLL spectral element method with $N=16$ and $N_{e}=2^{12}$. Figure 3 shows the relative error (with respect to the reference solution) of the first eigenvalue of the covariance kernel (18) for GLL elements of degree $1,2,3$ and 4 . The convergence rate does increase with $N$, as expected.

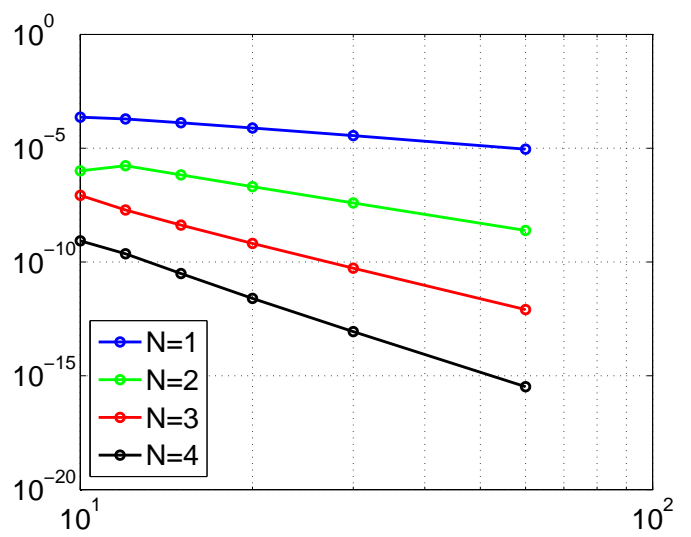

Figure 3. Relative GLL error the first eigenvalue versus number of elements for the Gaussian covariance kernel.

\section{CONCLUSIONS}

The spectral element method was employed to numerically compute the eigenvalues and eigenfunctions of the Fredholm integral equation (1). The use of Gauss-Lobatto-Legendre 
(GLL) collocation points and reduced integration with GLL quadrature rendered the implementation similar to piecewise constant finite elements in the sense that the resulting discrete equations are a standard eigenvalue problem rather than a generalized one. Preliminary numerical experiments show that the GLL spectral element method performs well in Fredholm eigenvalue problems.

\section{Acknowledgements}

This research is supported by Brazilian agency CNPq under grant 314553/2009-6, and by the Paraná state agency Fundação Araucária.

\section{REFERENCES}

[1] Azevedo J. S., Murad M. A., Borges M. R., Oliveira S. P. "A spacetime multiscale method for computing statistical moments in strongly heterogeneous poroelastic media of evolving scales". Int. J. Numer. Meth. Engrg., 2012, in press.

[2] Azevedo J. S. , Oliveira S. P. "A numerical comparison between quasi-Monte Carlo and sparse grid stochastic collocation methods". Commun. Comput. Phys. 12, 1051-1069, 2012.

[3] Castrilln-Cands J. H. and Amaratunga K. "Fast Estimation of Continuous KarhunenLoève Eigenfunctions Using Waveletss”. IEEE Trans. Signal Process. 50, 205-228, 2002.

[4] Frauenfelder P., Schwab C. and Todor R. "Finite elements for elliptic problems with stochastic coefficients". Comput. Meth. Appl. Mech. Engrg. 194, 205-228, 2005.

[5] Komatitsch D and Tromp J. "Introduction to the spectral-element method for 3-D seismic wave propagation”. Geophys. J. Int. 139, 806-822, 1999.

[6] Oliveira S. P. and Seriani G. "Effect of Element Distortion on the Numerical Dispersion of Spectral Element Methods". Commun. Comput. Phys. 9, 937-958, 2011.

[7] Tang T., Xu X and Cheng J. "On spectral methods for Volterra integral equations and the convergence analysis”. J. Comput. Math. 26, 825837, 2008. 